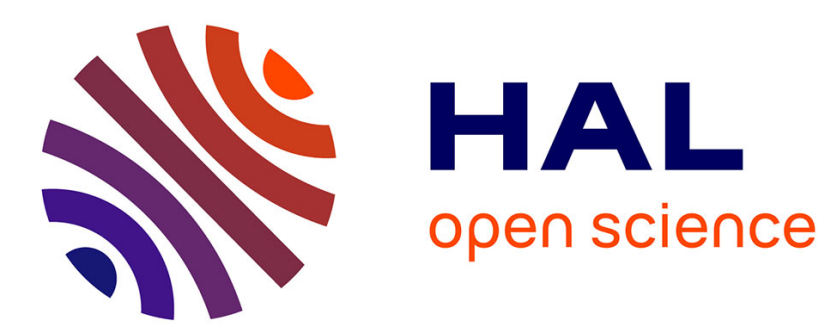

\title{
Theoretical study of gain distortions in dual-pump fiber optical parametric amplifiers
}

\author{
A. Vedadi, Arnaud Mussot, E. Lantz, H. Maillotte, T. Sylvestre
}

\section{To cite this version:}

A. Vedadi, Arnaud Mussot, E. Lantz, H. Maillotte, T. Sylvestre. Theoretical study of gain distortions in dual-pump fiber optical parametric amplifiers. Optics Communications, 2006, 267, pp.244-252. 10.1016/j.optcom.2006.05.074 . hal-00172283

\section{HAL Id: hal-00172283 \\ https://hal.science/hal-00172283}

Submitted on 28 Aug 2013

HAL is a multi-disciplinary open access archive for the deposit and dissemination of scientific research documents, whether they are published or not. The documents may come from teaching and research institutions in France or abroad, or from public or private research centers.
L'archive ouverte pluridisciplinaire HAL, est destinée au dépôt et à la diffusion de documents scientifiques de niveau recherche, publiés ou non, émanant des établissements d'enseignement et de recherche français ou étrangers, des laboratoires publics ou privés. 


\title{
Theoretical study of gain distortions in dual-pump fiber optical parametric amplifiers
}

\author{
Armand Vedadi *, Arnaud Mussot ${ }^{1}$, Eric Lantz, Hervé Maillotte, Thibaut Sylvestre \\ Département d'Optique P.M. Duffieux, Institut FEMTO-ST, Université de Franche-Comté, CNRS UMR 6174, F-25030 Besançon Cedex, France
}

Received 9 March 2006; received in revised form 25 May 2006; accepted 31 May 2006

\begin{abstract}
We study analytically and numerically the small signal gain in dual-pump fiber optical parametric amplifiers by including the phase modulation of the pump waves needed for practically increasing the stimulated Brillouin scattering threshold. As for the single-pump case, we show that large signal gain distortions are generated under co-phase modulation, which depend on the rise/fall time of the phase modulation and on the fiber dispersion slope. However, it is clearly confirmed that the counter-phase modulation scheme allows to efficiently suppress these gain distortions over the whole flat gain region. In addition, we demonstrate through realistic numerical simulations that this useful technique overcomes the additional impact of pump-phase modulation to amplitude modulation conversion and zero-dispersion wavelength variations.
\end{abstract}

(c) 2006 Published by Elsevier B.V.

Keywords: Fiber optical parametric amplifier; Four-wave mixing; phase modulation

\section{Introduction}

As well as being an optical amplifier, a fiber optical parametric amplifier (FOPA) is versatile and can be used for a variety of all-optical signal processing techniques for future ultra-fast optical networks such as wavelength conversion, optical multiplexing, sampling, limiting, switching, noise and dispersion monitoring [1-3]. In all FOPAs, it is necessary to employ schemes in order to avoid stimulated Brillouin scattering (SBS) of the high-power continuous-wave pumps. One approach is to broaden the Brillouin gain spectrum by varying parameters of the fiber such as temperature, strain, doping or geometrical properties [4-6]. The most commonly used approach, however, is to increase the SBS threshold by phase modulation of the pumps,

\footnotetext{
* Corresponding author.

E-mail addresses: armand.vedadi@univ-fcomte.fr (A. Vedadi), thibaut. sylvestre@univ-fcomte.fr (T. Sylvestre).

${ }^{1}$ Present address: Laboratoire de Physique des Lasers, Atomes et Molécules, Université des Sciences et Technologies de Lille, CNRS UMR 8523 , France.
}

which in turn induces other limitations on the FOPA performance. First, phase modulation has a detrimental effect on the coherently-coupled idler wave generated by fourwave mixing (FWM), by inducing its spectral broadening by twice that of the phase-modulated pump wave. Second, as it has been recently demonstrated theoretically [7], the phase modulation of the pump wave can induce large signal gain distortions which depend both on the rise/fall time of the phase modulator and on the dispersion slope of the amplifying fiber. Its impact on system performances has recently been observed through bit-error rate and Q penalties measurements $[8,9]$. Here we must stress that the latter effect is highly detrimental because it impairs all potential applications of FOPA-based optical devices.

To avoid the undesirable effects of phase modulation in FOPA-based wavelength converters, various techniques have been proposed and demonstrated [10-14]. Idler spectral broadening has been partially or totally cancelled by using either a binary-phase shift keying phase modulation or a dual-pumping out-of-phase scheme, respectively. In the latter case, the counter-phase modulation scheme was employed so that the frequency chirp induced on the idler 
wave by one pump is exactly balanced by an opposite frequency chirp induced by the second pump, paving the way for fully transparent parametric wavelength conversion [10]. The same suppression technique for the signal gain distortions induced by the pump-phase modulation has been recently suggested through simple considerations and quantified with an analytical expression $[9,15,16]$. The purpose of the present paper is to investigate this effect both analytically and numerically to clearly show the effectiveness of counter-phase modulation technique. To this end, we present a theoretical analysis of the small signal gain in a two-pump (2P) FOPA that fully takes into account the time-dependent phase of the pump waves. Our model is able to predict the large gain distortions induced by the phase modulation of the pump waves. As for the single-pump case, it is shown that these gain fluctuations depend both on the fiber dispersion slope and on the rise/fall time of the phase modulator. As in Refs. [9,15,16], our results show that these gain distortions can indeed be cancelled over the whole flat gain region when the two pump waves are counter-phase modulated. The results of our analytical approach are shown to be in good agreement with realistic numerical simulations of the non-linear Schrödinger equation with a NRZ $10 \mathrm{Gbit} / \mathrm{s}$ small signal and a pseudo-random bit sequence (PRBS) phase modulation for the pump waves. In addition, our numerical results show that the cancellation technique is robust against the additional impairment of phase modulation (PM) to amplitude modulation (AM) conversion of the pump waves, recently predicted by Yaman et al. [15] for 2P-FOPA. Finally, the influence of zero-dispersion wavelength (ZDW) variations on the impact of pump-phase modulation is also studied.

\section{Analytical model}

Parametric amplification driven by two pump waves in a silica fiber is based on non-degenerate four-wave mixing (FWM) whereby two pump waves at frequencies $\left(\omega_{1}, \omega_{2}\right)$ around the zero-dispersion wavelength (ZDW) amplify a frequency-detuned signal $\omega_{\mathrm{S}}$ and generate a phase-conjugated idler wave $\omega_{\mathrm{I}}$ [17]. Unlike for the single-pump case, the dual-pump case additionally leads to the generation of two sidebands $\left(\omega_{\mathrm{SB} 1}, \omega_{\mathrm{SB} 2}\right)$ that result from degenerate FWM of the signal and idler waves with one pump alone, as it is depicted on Fig. 1. Depending on the frequency detuning between the pumps and signal, these non-phase matched waves can have more and less influence on the parametric gain, by shrinking the gain bandwidth near the pump waves $[18,19]$. To assess the impact of pumpphase modulation on the parametric gain, let us first consider the total electric field that can be written as

$E(t, x, y, z)=A(t, z) F(x, y) \mathrm{e}^{-\mathrm{j}\left(\omega_{0} t-\beta_{0} z\right)}$,

where $\omega_{0}=\left(\omega_{1}+\omega_{2}\right) / 2$ is the mean pump frequency and $\beta_{0}=\beta\left(\omega_{0}\right)$ the propagation constant. $F(x, y)$ is the transverse field distribution and $A(t, z)$, which will be denoted

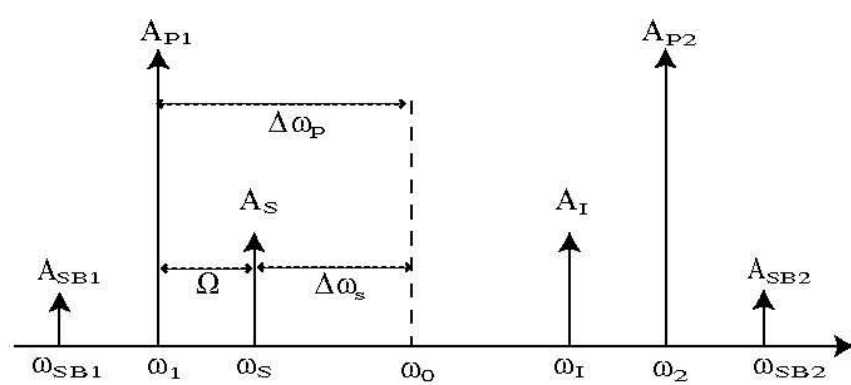

Fig. 1. Exact model of a two-pump fiber optical parametric amplifier.

$A$ for simplicity, is the slowly varying amplitude of the field. Taking into account the above mentioned interacting waves and neglecting $F(x, y)$ dependency on the frequency, $A$ can be expressed as

$A=A_{P_{1}}+A_{P_{2}}+A_{\mathrm{S}}+A_{1}+A_{\mathrm{SB} 1}+A_{\mathrm{SB} 2}$.

The wave amplitudes $A_{m}$ are connected to the Fourier transforms $A\left(\omega_{m}, z\right)$ by

$A_{m}=A\left(\omega_{m}, z\right) \mathrm{e}^{-\mathrm{j}\left[\left(\omega_{m}-\omega_{0}\right) t-\left(\beta\left(\omega_{m}\right)-\beta_{0}\right) z\right]}$,

$A$ is found to verify the non-linear Schrödinger equation (NLSE). Neglecting the fiber loss and including higher-order dispersion coefficients, NLSE takes the following form:

$\frac{\partial A}{\partial z}=\mathrm{j}\left\{\sum_{k=2}^{\infty} \mathrm{j}^{k} \frac{\beta_{k}}{k !} \frac{\partial^{k} A}{\partial \tau^{k}}+\gamma|A|^{2} A\right\}$,

where $\tau=t-z / v_{\mathrm{g}}$ is the time expressed in a reference frame moving at the group velocity of the mean pump frequency $\omega_{0} . \beta_{k}$ is the $k$ th-order dispersion coefficients, and $\gamma$ the non-linear coefficient. Inserting Eq. (2) in Eq. (4) and assuming that all amplitudes are negligible with respect to the pumps, we obtain the following equations for the pumps:

$$
\begin{aligned}
\frac{\partial A_{P_{i}}}{\partial z}= & \sum_{k=2}^{\infty} \mathrm{j} \frac{\beta_{k}}{k !}\left(\omega_{i}-\omega_{0}\right)^{k} A_{P_{i}} \\
& +\mathrm{j} \gamma\left(\left|A_{P_{i}}\right|^{2}+2\left|A_{P_{3-i}}\right|^{2}\right) A_{P_{i}} \quad(i=1,2) .
\end{aligned}
$$

Note also that we assume higher-order harmonics that could be generated from FWM of the two pump waves at frequencies $\left(2 \omega_{2}-\omega_{1}, 2 \omega_{1}-\omega_{2}\right)$ are negligible. For the signal wave $A_{\mathrm{s}}$, Eq. (4) leads to the following equation:

$$
\begin{aligned}
\frac{\partial A_{\mathrm{S}}}{\partial z}= & \mathrm{j}\left(\sum_{k=2}^{\infty} \frac{\beta_{k}}{k !}\left(\omega_{\mathrm{S}}-\omega_{0}\right)^{k}+2 \gamma\left(\left|A_{P_{1}}\right|^{2}+\left|A_{P_{2}}\right|^{2}\right)\right) A_{\mathrm{S}} \\
& +2 \mathrm{j} \gamma A_{P_{1}} A_{P_{2}} A_{1}^{*}+2 \mathrm{j} \gamma A_{P_{1}} A_{P_{2}}^{*} A_{\mathrm{SB} 2}+2 \mathrm{j} \gamma A_{P_{1}}^{2} A_{\mathrm{SB} 1}^{*} .
\end{aligned}
$$

Similar equations can be easily derived for the idler wave $A_{\mathrm{I}}$, and the non-phase matched waves $A_{\mathrm{SB} 1}$ and $A_{\mathrm{SB} 2}$. Now, we assume a time-dependent phase $\varphi_{i}(\tau)$ that accounts for the phase modulation of the two pump waves. This phase term will indeed induce an instantaneous frequency chirp on both pump waves that can be approximated by $\omega_{i}(\tau)=\omega_{i}+\varphi_{i, \tau}$, with $\varphi_{i, \tau}=\frac{\partial \varphi_{i}(\tau)}{\partial \tau}$ the first-order time derivative of the phase. The phase matching conditions for all parametric processes will be modified, thus shifting the idler and sidebands frequencies, 


$$
\begin{aligned}
& \omega_{1}^{\prime}=\omega_{1}+\varphi_{1, \tau}+\varphi_{2, \tau}, \\
& \omega_{\mathrm{SB} 1}^{\prime}=\omega_{\mathrm{SB} 1}+2 \varphi_{1, \tau}, \\
& \omega_{\mathrm{SB} 2}^{\prime}=\omega_{\mathrm{SB} 2}+\varphi_{2, \tau}-\varphi_{1, \tau} .
\end{aligned}
$$

It is also convenient to make the following phase rotation of the field variables

$$
\begin{aligned}
& A_{i}^{\prime}=A_{i} \mathrm{e}^{-\mathrm{j} \sum_{k=}^{\infty}(-1)^{k \frac{\beta_{k}}{k !}\left(\Delta \omega_{\mathrm{P}}+\varphi_{1, \tau}\right)^{k} z}} \quad\left(i=P_{1}, \mathrm{~S}, \mathrm{SB} 1\right), \\
& A_{i}^{\prime}=A_{i} \mathrm{e}^{-\mathrm{j} \sum_{k=}^{\infty} \frac{\beta_{k}}{k !}\left(\Delta \omega_{\mathrm{P}}+\varphi_{2, \tau}\right)^{k_{z}}} \quad\left(i=P_{2}, \mathrm{I}, \mathrm{SB} 2\right),
\end{aligned}
$$

where $\Delta \omega_{\mathrm{P}}=\omega_{2}-\omega_{0}$ is the pump frequency detuning. Thus, Eq. (5) rewrites

$\frac{\partial A_{P_{i}}^{\prime}}{\partial z}=\mathrm{j} \gamma\left(\left|A_{P_{i}}^{\prime}\right|^{2}+2\left|A_{P_{3-i}^{\prime}}\right|^{2}\right) A_{P_{i}}^{\prime} \quad(i=1,2)$.

In the undepleted pump approximation, Eq. (9) admits the following steady-state CW solutions for the two pump waves

$A_{P_{i}}^{\prime}=\sqrt{P_{i}} \exp \left(\mathrm{j} \gamma\left(P_{i}+2 P_{3-i}\right) z\right) \quad(i=1,2)$,

where $P_{i}$ is the power of the pump $i(i=1,2)$ at the fiber input. Substituting Eq. (10) and (8) into Eq. (6), one gets for the signal wave $A_{\mathrm{S}}^{\prime}$

$$
\begin{aligned}
\frac{\partial A_{\mathrm{S}}^{\prime}}{\partial z}= & \sum_{k=2}^{\infty} \mathrm{j} \frac{\beta_{k}}{k !}(-1)^{k}\left[\Delta \omega_{\mathrm{S}}^{k}-\left(\Delta \omega_{\mathrm{P}}-\varphi_{1, \tau}\right)^{k}\right] A_{\mathrm{S}}^{\prime} \\
& +2 \mathrm{j} \gamma\left(P_{1}+P_{2}\right) A_{\mathrm{S}}^{\prime}+2 \mathrm{j} \gamma r A_{1}^{\prime *} \mathrm{e}^{3 \mathrm{j} \gamma\left(P_{1}+P_{2}\right) z} \\
& +2 \mathrm{j} \gamma r A_{\mathrm{SB} 2}^{\prime} \mathrm{e}^{\mathrm{j} \gamma\left(P_{2}-P_{1}\right) z}+2 \mathrm{j} \gamma P_{1} A_{\mathrm{SB} 1}^{\prime *} \mathrm{e}^{2 \mathrm{j} \gamma\left(P_{1}+2 P_{2}\right) z},
\end{aligned}
$$

where $r=2 \gamma \sqrt{P_{1} P_{2}}$ and $\Delta \omega_{\mathrm{S}}=\omega_{\mathrm{I}}-\omega_{0}$ is the signal frequency detuning. Similar equations can be also derived for $A_{1}^{\prime}, A_{\mathrm{SB} 1}^{\prime}$ and $A_{\mathrm{SB} 2}^{\prime}$. A second transformation of the fields is necessary in order to eliminate the $z$ dependence

$$
\begin{array}{ll}
A_{i}^{\prime \prime}=A_{i}^{\prime} \mathrm{e}^{-\mathrm{j} \gamma\left(P_{1}+2 P_{2}\right) z} & (i=\mathrm{S}, \mathrm{SB} 1), \\
A_{i}^{\prime \prime}=A_{i}^{\prime} \mathrm{e}^{-\mathrm{j} \gamma\left(2 P_{1}+P_{2}\right) z} & (i=\mathrm{I}, \mathrm{SB} 2) .
\end{array}
$$

Inserting Eq. (12) in Eq. (11), we obtain a set of four equations that can be written as

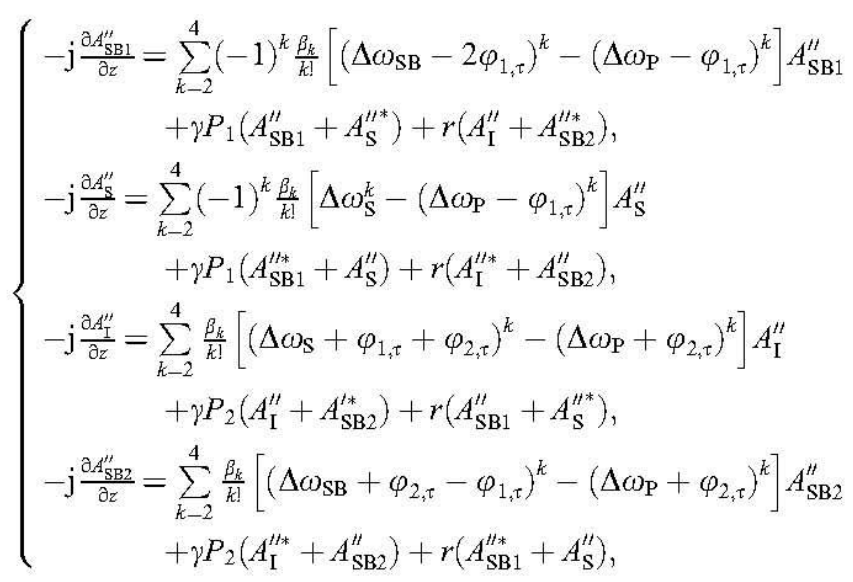

with $\Delta \omega_{\mathrm{SB}}=2 \Delta \omega_{\mathrm{P}}-\Delta \omega_{\mathrm{S}}$. The parametric gain is then extracted by solving numerically the above set of equations. Nevertheless, a straightforward analytical formula of the parametric gain can be derived by taking only into account the signal and the idler waves. Note that this truncated model is valid only for signal and idler far enough from the pumps, so that the impact of the sidebands $\omega_{\mathrm{SB} 1}$ and $\omega_{\mathrm{SB} 2}$ on the parametric gain becomes negligible [18]. The set of equations therefore reduces to

$$
\left\{\begin{aligned}
-\mathrm{j} \frac{\partial A_{\mathrm{S}}^{\prime \prime}}{\partial z}= & \left(\sum_{k=2}^{4}(-1)^{k} \frac{\beta_{k}}{k !}\left[\Delta \omega_{\mathrm{S}}^{k}-\left(\Delta \omega_{\mathrm{P}}-\varphi_{1, \tau}\right)^{k}\right]+\gamma P_{1}\right) A_{\mathrm{S}}^{\prime \prime}+r A_{\mathrm{I}}^{\prime \prime *} \\
-\mathrm{j} \frac{\partial A_{\mathrm{I}}^{\prime \prime}}{\partial z}= & \left(\sum _ { k = 2 } ^ { 4 } \frac { \beta _ { k } } { k ! } \left[\left(\Delta \omega_{\mathrm{S}}+\varphi_{1, \tau}+\varphi_{2, \tau}\right)^{k}\right.\right. \\
& \left.\left.-\left(\Delta \omega_{\mathrm{P}}+\varphi_{2, \tau}\right)^{k}\right] A_{\mathrm{I}}^{\prime \prime}+\gamma P_{2}\right) A_{\mathrm{I}}^{\prime \prime}+r A_{\mathrm{S}}^{\prime \prime *} .
\end{aligned}\right.
$$

The parametric gain $g$ for this truncated model takes the following form:

$g=\sqrt{4 \gamma^{2} P_{1} P_{2}-\left(\frac{\kappa+\delta \kappa}{2}\right)^{2}}$,

where $\kappa$ is the standard phase mismatch

$\kappa=\gamma\left(P_{1}+P_{2}\right)+\beta_{2}\left(\Delta \omega_{\mathrm{S}}^{2}-\Delta \omega_{\mathrm{p}}^{2}\right)+\frac{\beta_{4}}{12}\left(\Delta \omega_{\mathrm{S}}^{4}-\Delta \omega_{\mathrm{p}}^{4}\right)$

and $\delta \kappa$ the instantaneous phase mismatch due to pumpphase modulation. Assuming that $\left|\varphi_{i, \tau}\right| \ll\left(\Delta \omega_{P}-\Delta \omega_{\mathrm{S}}\right)$, which is valid for common shifts used in phase modulation techniques, $\delta \kappa$ can be simplified to

$\delta \kappa=\frac{\beta_{3}}{2}\left(\Delta \omega_{\mathrm{S}}^{2}-\Delta \omega_{\mathrm{P}}^{2}\right)\left(\varphi_{1, \tau}+\varphi_{2, \tau}\right)$.

Eqs. (15) and (17) together show that the parametric gain depends now both on the fiber dispersion slope and on the frequency chirp induced by the pump-phase modulation. Note that if we set $\delta \kappa=0$ in (15), we retrieve the usual expression of the parametric gain for the case of non-modulated pumps [17]. Note also that the instantaneous phase mismatch has a negligible dependency on $\beta_{2}$ and $\beta_{4}$. We can also readily deduce from Eq. (15) that the gain becomes time-independent again when the two pumps are in phase opposition, i.e., $\varphi_{1, \tau}=-\varphi_{2, \tau}$, as expected. Finally, the net signal gain at the 2P-FOPA's output with length $L$ is given by

$G=1+\left(\frac{2 \gamma \sqrt{P_{1} P_{2}}}{g} \sinh (g L)\right)^{2}$.

To demonstrate the cancellation technique, we study and compare two 2P-FOPA configurations based on a $300 \mathrm{~m}$ long highly non-linear fiber (HNLF) with $\beta_{3}=$ $1.2 \times 10^{-40} \mathrm{~s}^{3} \mathrm{~m}^{-1}, \quad \beta_{4}=2.85 \times 10^{-55} \mathrm{~s}^{4} \mathrm{~m}^{-1}$ and $\gamma=18$ $\mathrm{W}^{-1} \mathrm{~km}^{-1}$. HNLFs indeed allow for the achievement of ultra-wide and flat parametric gain band in the $1.55 \mu \mathrm{m}$ region, depending on an accurate tuning of the pump frequency separation $\Delta \omega_{\mathrm{P}}$ with respect to both $\beta_{2}$ and $\beta_{4}$ dispersion coefficients. The first $2 \mathrm{P}$-FOPA has a mean pump frequency at the exact ZDW in order to achieve a flat $45 \mathrm{~nm}$ $(5.6 \mathrm{THz})$ gain bandwidth. The second 2P-FOPA has been optimized to generate an ultrawide $74 \mathrm{~nm}(9.2 \mathrm{THz})$ 
bandwidth with ripples of less than $0.2 \mathrm{~dB}$, using the Chebyshev polynomial method of Ref. [17]. Note that a relatively high value of $\beta_{3}$ was chosen compared to standard HNLFs in order to stress the impact of phase modulation on gain distortions. Nevertheless, this value is standard for conventional dispersion shifted fibers (DSFs), which have shown to be good candidates for 2P-FOPAs in terms of crosstalk for WDM systems [20]. In addition, we assume that the two pumps are $\pi$-binary phase-shift-keying phase-modulated by a pseudo-random-bit sequence (PRBS) operating at a frequency of $3-\mathrm{GHz}$ and with a realistic rise-fall time of $27-p s$. This modulation scheme induces a uniform broadening of the pumps spectra by the frequency modulation and has been demonstrated for both efficient SBS suppression and idler spectral broadening reduction [14]. Fig. 2(a) depicts one bit of the phase sequence (solid line) and its associated time derivative (dashed line). Figs. 3(a) and (b) show the instantaneous parametric gain spectrum of $2 \mathrm{P}$-FOPAs for the two extreme values of the phase derivative $\varphi_{1, \tau}= \pm 1 \times$ $10^{11} \mathrm{rad} \mathrm{s}^{-1}$ when both pumps are in phase, and without phase modulation (solid line), derived from Eq. (18) of the truncated model. For comparison, the parametric gain band obtained from the exact solutions of (Eq. (13)) are also plotted in Figs. 3(c) and (d). First, we see on Figs. 3(a)-(d) noticeable deterioration of the gain spectrum for both extrema of the frequency chirp, meaning that the flatness of the $2 \mathrm{P}$ FOPA is not conserved during each phase jump. Indeed, the phase modulation of the pump waves induces an instantaneous pump frequency chirp (or dithering) that will modify the phase-matching condition through the term $\delta \kappa$ in Eq. (17), and subsequently the parametric gain. This detrimental effect is so much marked in the second FOPA configuration
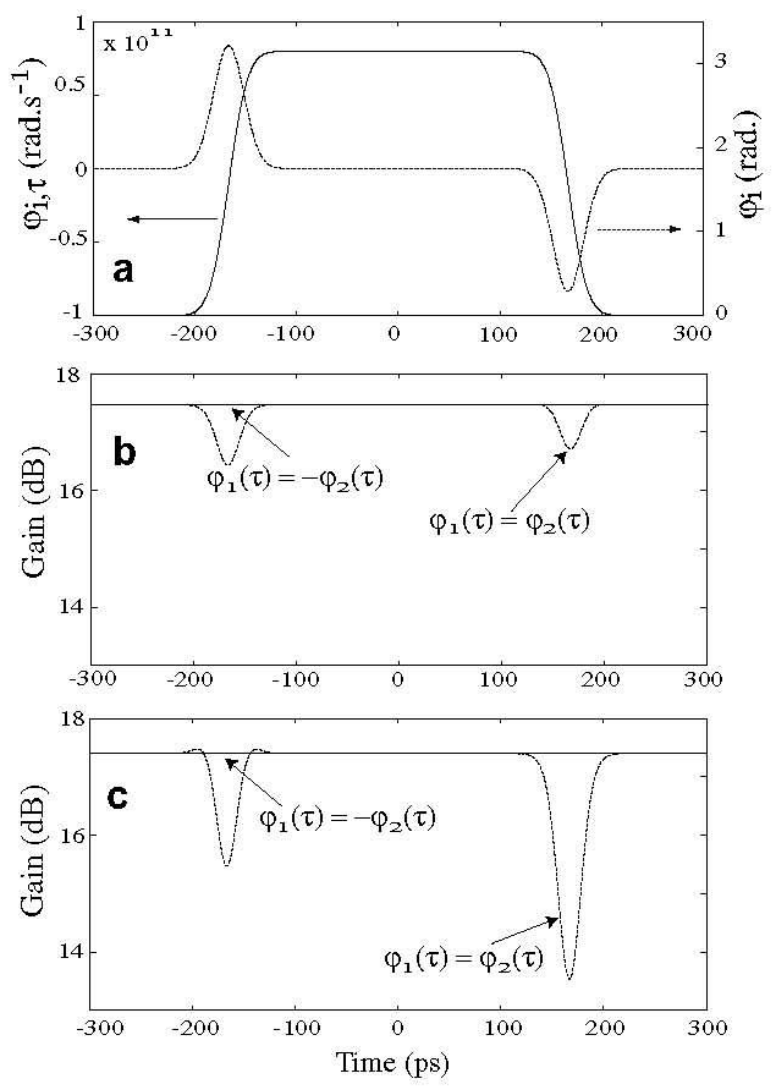

Fig. 2. (a) Phase of the pump waves (solid line, right scale) and its associated time derivative (dashed line, left scale). ( $b$, c) Instantaneous parametric gain of a CW signal located at $\Delta \omega_{\mathrm{S}}=0.5 \mathrm{THz}$ for the two 2P-FOPAs (dashed lines: $\varphi_{1}=\varphi_{2}$ ), (solid lines: $\varphi_{1}=-\varphi_{2}$ ). Parameters are (b) $\beta_{2}=0$, (c) $\beta_{2}\left(\omega_{0}\right)$ $=-2 \times 10^{-29} \mathrm{~s}^{2} \mathrm{~m}^{-1}, \beta_{3}=1.2 \times 10^{-40} \mathrm{~s}^{3} \mathrm{~m}^{-1}, \beta_{4}=2.85 \times 10^{-55} \mathrm{~s}^{4} \mathrm{~m}^{-1}$, $\gamma=18 \mathrm{~W}^{-1} \mathrm{~km}^{-1}, P_{1}=P_{2}=250 \mathrm{~mW}$ and $L=300 \mathrm{~m}$.

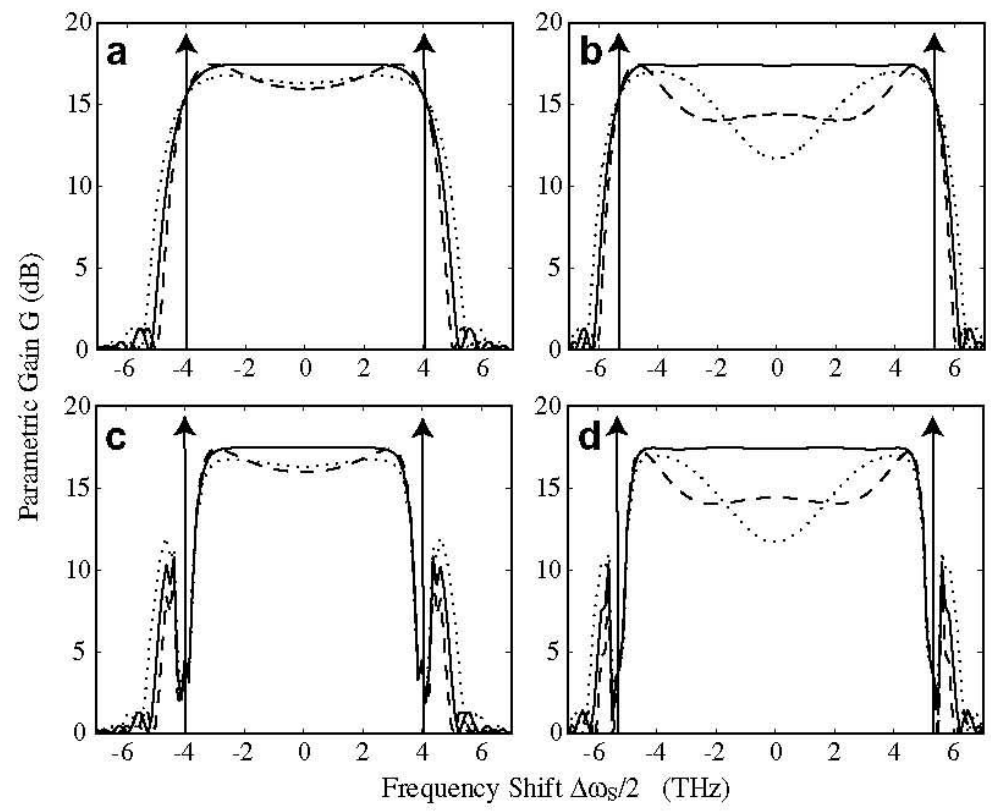

Fig. 3. Parametric gain of the two 2P-FOPAs during the rise time (dashed lines) and the fall time (dotted lines) of the phase modulation when $\varphi_{1}=\varphi_{2}$ and without phase modulation (solid lines), with the truncated model $(a, b)$, and with exact solutions (c,d). Pump frequency detunings are ( $a, c)$ $\Delta \omega_{\mathrm{P}}=2 \pi \times 4 \mathrm{THz},(\mathrm{b}, \mathrm{d}) \Delta \omega_{\mathrm{P}}=2 \pi \times 5.3 \mathrm{THz}$. 
that the signal gain exhibits a strong gap at the center of the band. Note also that in all cases the maximum parametric gain is lowered during both the rise time and the fall time of the phase modulation. Second, the direct comparison between Figs. 3(a) and (b) and Figs. 3(c) and (d) shows that the gain spectrum has significantly shrunk near the pump frequencies when the two external sidebands $A_{\mathrm{SB} 1}$ and $A_{\mathrm{SB} 2}$ generated by FWM are taken into account. These waves indeed are not phase-matched and therefore reduce the gain bandwidth, as it has been previously demonstrated numerically [18]. This comparison indicates that the standard model of FWM is incomplete and gives incorrect results when the signal frequency detuning $\Delta \omega_{\mathrm{S}}$ is comparable to the pump frequency detuning $\Delta \omega_{\mathrm{P}}$. In Fig. 2(b) and (c) is illustrated the impact of the pump-phase modulation on the instantaneous parametric gain for a signal located at $\Delta \omega_{\mathrm{S}}=0.5 \mathrm{THz}$ for both 2P-FOPA configurations. As it can be seen, the phase modulation of the two pump waves induces large gain distortions during both the leading and the falling edges of the phase. Unlike for the single-pump case [7], the parametric gain is always reduced by the phase modulation in the dualpumps case, in good accordance with the gain spectra plotted in Fig. 3. In the worst case, the gain drops by more than $3 \mathrm{~dB}$ as shown by the dotted curve in Fig. 2(c). When the pumpphases have opposite signs, i.e., $\varphi_{1}=-\varphi_{2}$, the gain distortions are totally cancelled, as shown by the solid lines of Fig. 2(b) and (c).

\section{Numerical simulations}

To further study the impact of phase modulation on the parametric gain, we perform numerical simulations of the
2P-FOPAs with a numerical integration of NLSE Eq. (4). We consider the parametric amplification of a nonreturn-to-zero (NRZ) data modulation format at a bit rate of $R=10 \mathrm{Gbit} / \mathrm{s}$, in the same operating conditions as in Section 2. The two pump waves are phase-modulated with a $2^{7}-1$ PRBS sequence operating at $3-\mathrm{GHz}$ with common rise/fall time of $27 \mathrm{ps}$. The small signal is shifted by $\Delta \omega_{\mathrm{S}}=0.5 \mathrm{THz}$ from the mean pump frequency. Additionally, the receiver is modelled in a realistic manner as in Ref. [7]. We used an optical Fabry-Pérot filter (bandwidth $=4 R$ ) combined with an electrical square detector and a second-order Butterworth filter (bandwidth $=0.8 R$ ) [21]. Note also that no noise source term has been added in the simulation. We then compare the system performances of our two broadband and flat amplifiers studied in Section 2. The results of our numerical simulations are presented in Fig. 4(a) and (b) that show the output amplified NRZ signal when the two pumps are co-phase modulated. The phase of both pumps are also plotted in dashed lines in Fig. 4(a) and (b). Clearly, the signal undergoes sharp distortions during each phase jump. These results are in quite good agreement with the analytical study of Section 2. As an example, for the first 2P-FOPA configuration (Fig. 4(a)), the gain is lowered by $17 \%(0.8 \mathrm{~dB})$ and $12.5 \%(0.6 \mathrm{~dB})$ on a rising and a falling edge, respectively, in accordance with the analytical results of Fig. 2 (1 and $0.8 \mathrm{~dB}$ respectively). Figs. 4 (c) and (d) illustrate the electrical traces after the bandpass filter. The distortions seen on the NRZ signal are lowered because of the bandpass filtering, but they still remain present. It is important to emphasize that such ultra-fast signal distortions may be difficult to observe directly in a practical system. This is why indi-
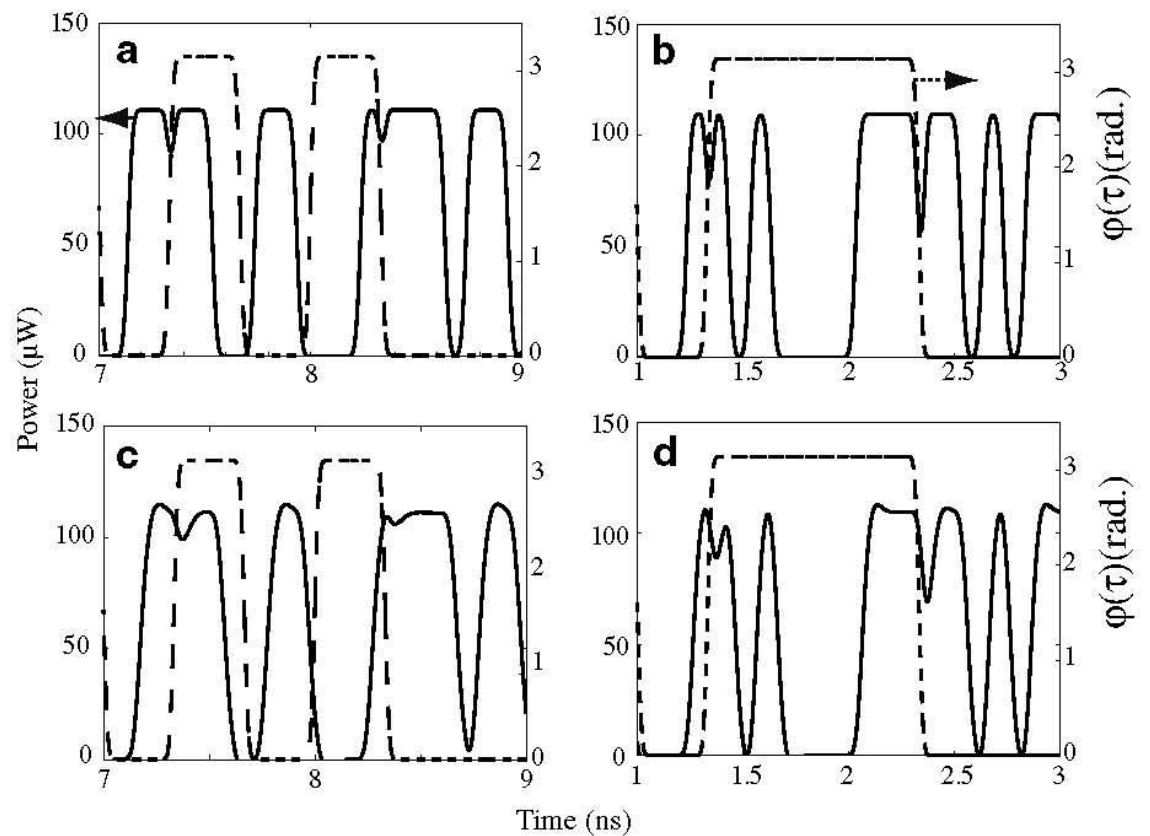

Fig. 4. (a,b) Optical, and $(c, d)$ electrical traces showing how a bit sequence is deteriorated by the phase modulation when the two pumps are in-phase. Dashed line, phase of the pumps. The input signal power is $2 \mu \mathrm{W}$. 
rect observations have been performed recently through bit-error rate and $\mathrm{Q}$ penalties measurements [8,9]. When the two pumps are synchronously counter-phase modulated, as illustrated on Fig. 5, the optical and electrical traces show that the signal distortions are almost cancelled, in good agreement with the analytical prediction of Section 2. Other numerical results, presented in Figs. 6(a)-(d), show the eye patterns at the FOPA's input and output when the pump are co-phase and counter-phase modulated, respectively. As it can be seen, the eye pattern is severely distorted at the FOPA's output, especially with the second 2P-FOPA under study (Fig. 6(c)), while it becomes as open as at the amplifier's input when counter-phase modulation is used. The residual small signal distortions seen in Fig. 5 can be attributed to additional impairment of the phase modulation (PM) to amplitude modulation (AM)
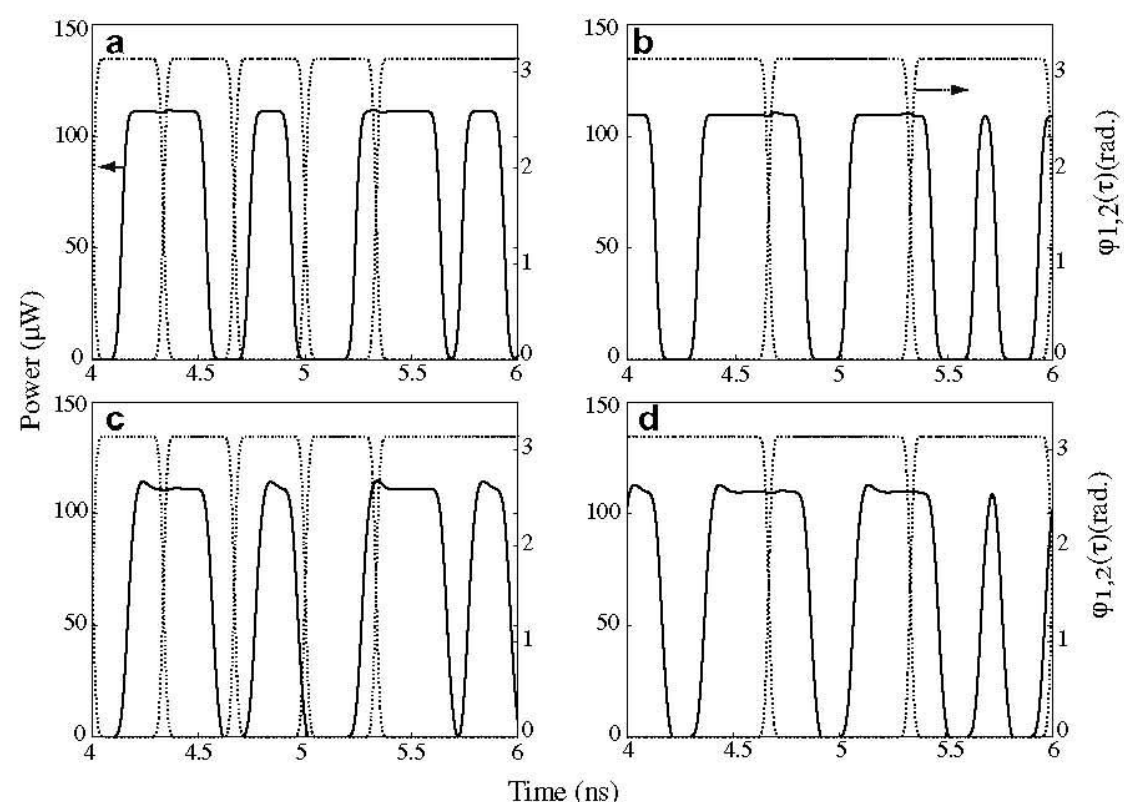

Fig. 5. $(\mathrm{a}, \mathrm{b})$ Optical, and $(\mathrm{c}, \mathrm{d})$ electrical traces showing how the signal distortions can be cancelled when the two pumps are out-of-phase. Dashed line, phase of the pumps.

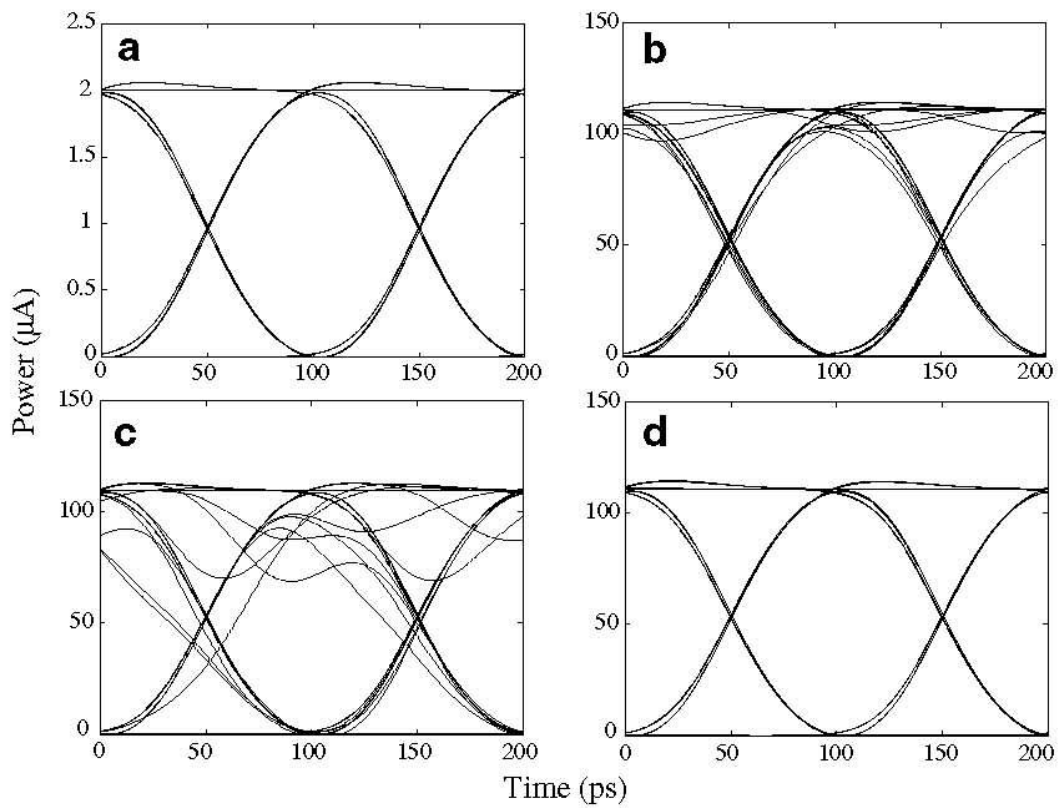

Fig. 6. Electrical eye patterns of the signal located at $0.5 \mathrm{THz}$ from $\omega_{0}$, (a) Amplifier's input. (b, c) Output of first and second 2P-FOPA under test respectively, when the pumps are in-phase modulated. (d) Amplifier's output when the pumps are out-of-phase modulated. 


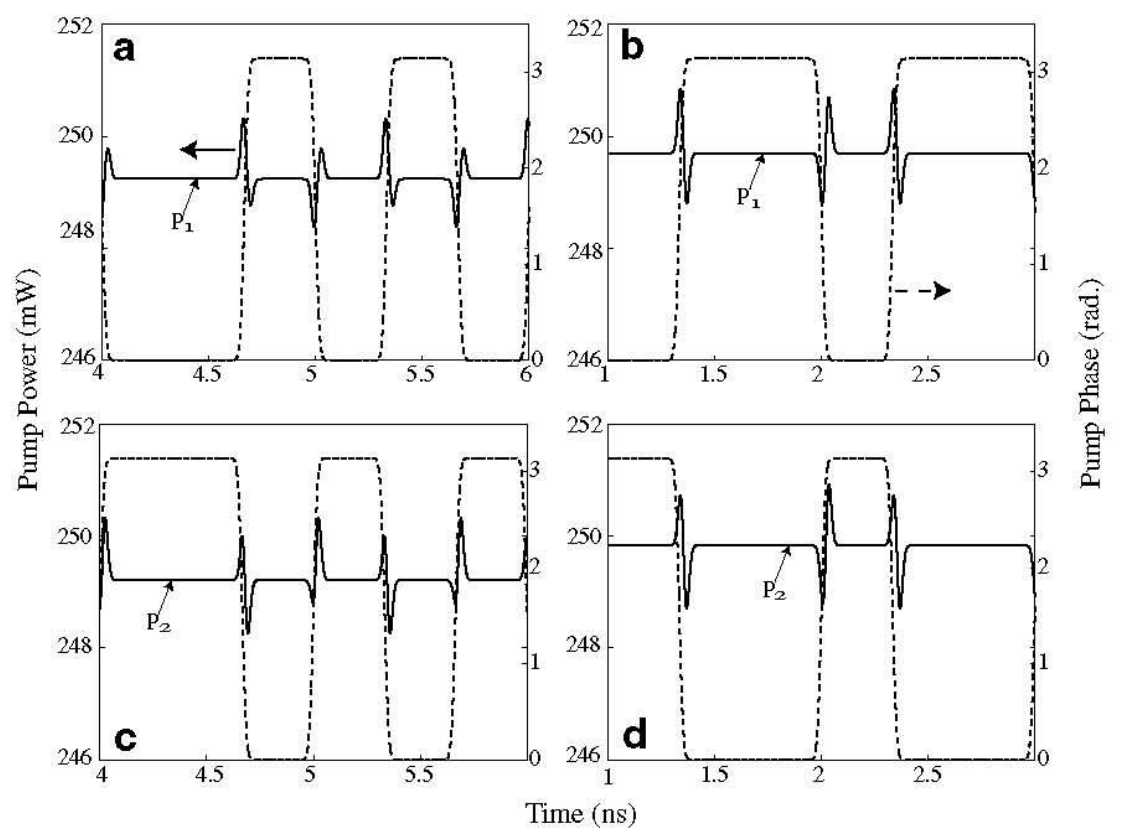

Fig. 7. Pump powers $\left(P_{1}, P_{2}\right.$, solid lines) and their phases $\left(\varphi_{1}, \varphi_{2}\right.$, dashed lines) at the FOPA output showing the small PM to AM conversion with the same parameters as in Fig. 2. (a,c) 1st 2P-FOPA, (b, d) 2nd 2P-FOPA.

conversion because of fiber dispersion, recently predicted for FOPA by Yaman et al. [15]. As demonstrated theoretically, the PM to AM conversion also leads to signal distortions via the parametric gain, even when a counter-phase modulation scheme is implemented. We numerically investigated the impact of PM to AM conversion on both the pump waves and the signal gain using NLSE integration. Fig. 7(a)-(d) shows the temporal evolution of the pump powers $\left(P_{1}, P_{2}\right.$, solid lines) and their phases $\left(\varphi_{1}, \varphi_{2}\right.$, dashed lines) at the FOPA's output for both 2P-FOPA configurations. We can see the PM to AM conversion of the pump waves which exhibit opposite amplitude modulations during a rise/fall time of the phase modulation because of their opposite group-velocity dispersion with respect to the ZDW. Consequently, the PM to AM conversion for the two pump waves cannot balance each other out to equalize the parametric gain. However, on Fig. 7(a)-(d), the PM to AM conversion induces very low pump power fluctuations, of less than $0.5 \%$ of the total continuous pump power. This numerical result clearly shows that the counter-phase pumping scheme does not suffer from PM to AM conversion of the pump waves for realistic rise/fall time commonly used to efficiently suppress SBS. However, because of its dependency on the second derivative of the pumps phase and on the pumps dispersion, PM to AM conversion can be detrimental for sharp rise/fall time of pump-phase modulation or wide amplifier bandwidth [15].

\section{Influence of dispersion fluctuations}

In practice, the $\mathrm{ZDW}$ of single-mode optical fibers longitudinally fluctuates due to random variations in opto-geometric parameters of the preform and drawing processes during fabrication. Because of the phase-matching conditions of FOPAs, the ZDW variations induce parametric gain ripple and reduce the gain bandwidth
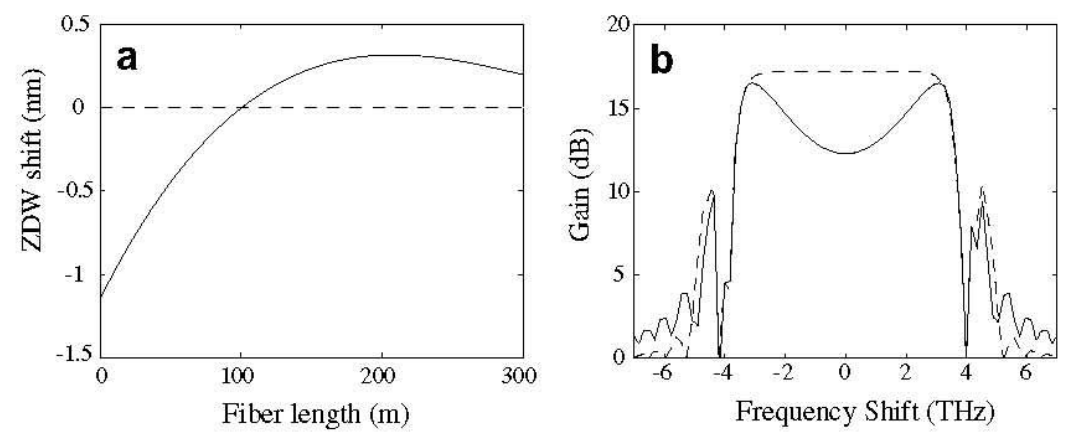

Fig. 8. (a) Zero-dispersion wavelength (ZDW) fluctuations map (solid line) and mean ZDW (dashed line). (b) Parametric gain spectrum of the 2P-FOPAs with $Z D W$ variations (solid line) and without $Z D W$ variations (dashed line). $\Delta \omega_{\mathrm{P}}=2 \pi \times 4 \mathrm{THz}$. 

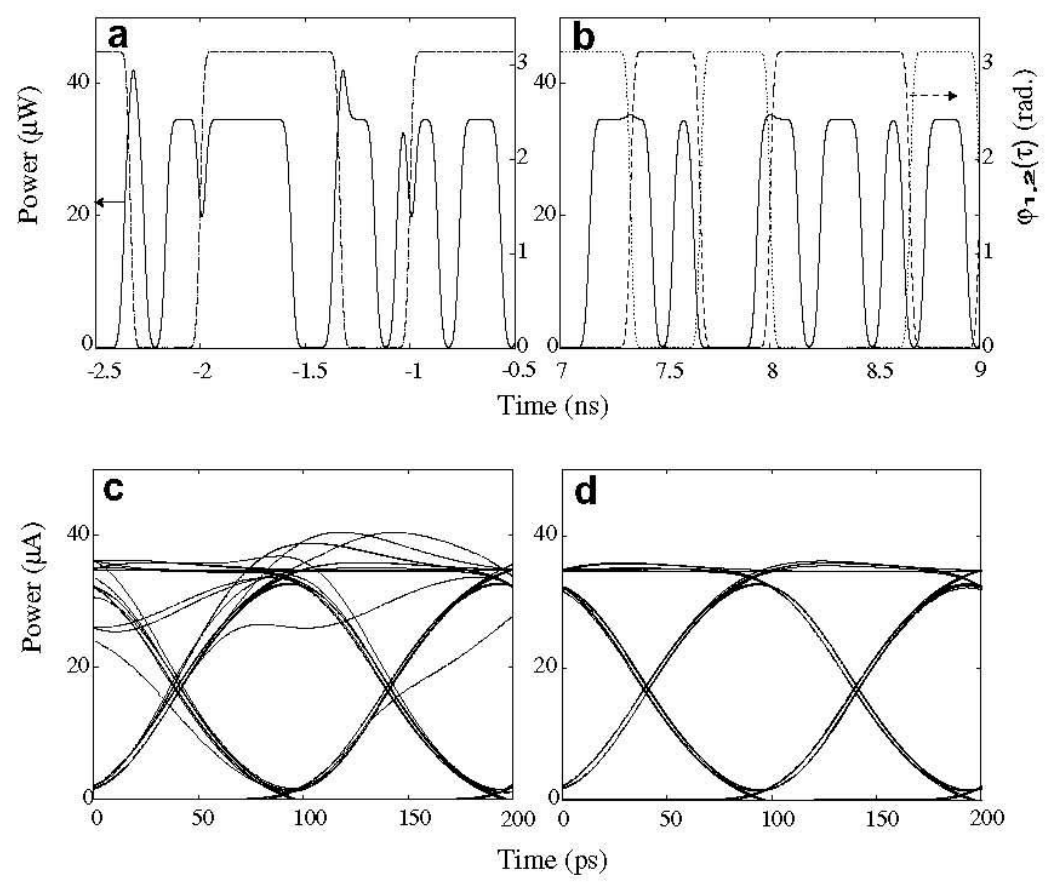

Fig. 9. (a,c) Optical trace and electrical eye pattern of a signal at the output of the 2P-FOPA with ZDW fluctuations when the two pumps are in-phase modulated, as indicated by the dashed lines, and $(b, d)$ out-of-phase modulated.

$[22,23]$. It has been shown in Ref. [9] that the ZDW fluctuations can strongly reduce $Q$ penalties caused by pump-phase modulation in FOPAs. While it is foreseeable from Eqs. (15)-(18) that the counter-phase modulation technique should remain robust against ZDW fluctuations, the fact that the optimized gain is not achieved in this case may lead to behaviors different of the last section. It is therefore important to assess the gain distortions due to pump-phase modulation with random fluctuations of ZDW. We therefore consider a $300-\mathrm{m}$ long HNLF whose ZDW variations was mapped in Ref. [24], as plotted in Fig. 8(a). In this section, we restrict our study to the first 2P-FOPA configuration of Sections 2 and 3 for which the mean pump frequency is exactly at the ZDW. Fig. 8(b) shows the gain spectrum obtained through a numerical integration of the NLSE with the same parameters as in Fig. 2(b). We then perform numerical simulations of a FOPA with a small signal located at $\Delta \omega_{\mathrm{S}}=0.5 \mathrm{THz}$ from the mean pump frequency. As shown in Fig. 9(a), when the two pumps are co-phase modulated, the signal undergoes substantial distortions due to pump-phase modulation. In particular, in addition to dips, one can also see spikes on the falling edges of the pumps phases. Unlike the former case of Section 3 when ZDW was considered constant and $\delta \kappa$ could only induce a gain decrease, the pump-phase shift may in some case enhance the phase-matching conditions thus increasing the instantaneous signal gain. When the counter-phase modulation is used, the temporal optical trace and the electrical eye diagrams of Figs. 9(b) and (d) show that the gain distortions are almost cancelled, meaning that the counter-phase modulation technique is robust against ZDW variations.

\section{Conclusion}

In this work, we have revisited theoretically the small signal gain of a broadband and flat dual-pump fiber optical parametric amplifier by including the phase modulation of the pumps, which is implemented in practice to avoid stimulated Brillouin back-scattering. It has been shown both analytically and numerically that the dualpumping counter-phased scheme in parametric amplifiers can in principle totally cancel not only the idler spectral broadening, but also the large gain distortions induced by pump-phase modulation. Moreover, our numerical results have shown that this useful technique does not significantly suffer from the additional impairment of pumpphase modulation to amplitude modulation conversion. Our results suggest that the dual-pumping counter phased scheme in parametric amplifiers is clearly more suitable for practical applications than single-pump scheme, as long as phase modulation is used to suppress stimulated Brillouin scattering.

\section{Acknowledgements}

The authors thank the Conseil Régional de FrancheComté for financial support and A. Durécu from Alcatel for helpful discussions. 


\section{References}

[1] J. Hansryd, P.A. Andrekson, M. Westlund, J. Lie, P.-O. Hedekvist, IEEE J. Select. Topics Quant. Electron. 8 (2002) 506.

[2] L. Provino, A. Mussot, E. Lantz, T. Sylvestre, H. Maillotte, J. Opt. Soc. Am. B 20 (7) (2003) 1532.

[3] T.T. Ng, J.L. Blows, J.T. Mok, R.W. McKerracher, B.J. Eggleton, J. Lightwave Technol. 23 (2005) 818.

[4] K. Tsujikawa, K. Nakajima, Y. Miyajima, M. Ohashi, IEEE Photon. Technol. Lett. 10 (1998) 1139.

[5] J. Hansryd, F. Dross, M. Westlund, P.A. Andrekson, S.N. Knudsen, J. Lightwave Technol. 19 (2001) 1691

[6] J. Marconi, IEE Electron. Lett. 40 (2004) 1522.

[7] A. Mussot, A. Durécu-Legrand, E. Lantz, C. Simmoneau, D. Bayart, H. Maillotte, T. Sylvestre, IEEE Photon. Technol. Lett. 16 (2004) 1289.

[8] A. Durécu-Legrand, A. Mussot, C. Simmoneau, D. Bayart, T. Sylvestre, E. Lantz, H. Maillotte, Electron. Lett. 41 (2005) 83.

[9] J.C. Boggio, A. Guimarães, F. Callegari, J. Marconi, H. Fragnito, Opt. Commun. 249 (2005) 451.

[10] M.C. Ho, M.E. Marhic, K.K.Y. Wong, L.G. Kazovsky, J. Lightwave Technol. 20 (2002) 469.

[11] K.K.Y. Wong, M.E. Marhic, L.G. Kasovsky, IEEE Photon. Technol. Lett. 15 (2003) 33.

[12] S. Radic, C.J. McKinstrie, R. Jopson, J.C. Centanni, A.R. Chraplyvy, C.G. Jorgensen, K. Brar, C. Headley, IEEE Photon. Technol. Lett. 15 (2003) 673 .
[13] S. Radic, R.M. Jopson, A. Gnauck, C.J. McKinstrie, J.C. Centanni, A.R. Chraplyvy, Stimulated Brillouin scattering suppression using a single modulator in two-pump parametric architectures, in: Optical Fiber Communications, No. OWN5, Anaheim, California, 2005.

[14] T. Tanemura, H.C. Lim, K. Kikuchi, IEEE Photon. Technol. Lett. 13 (2001) 1328.

[15] F. Yaman, Q. Lin, S. Radic, G.P. Agrawal, IEEE Photon. Technol. Lett. 17 (2005) 2053.

[16] P. Kylemark, M. Karlsson, P.A. Andrekson, IEEE Photon. Technol. Lett. 18 (2006) 439.

[17] M.E. Marhic, Y. Park, F.S. Yang, L.G. Kazovsky, Opt. Lett. 21 (1996) 1354.

[18] C.J. McKinstrie, S. Radic, Opt. Lett. 27 (2002) 1138

[19] E. Lantz, D. Gindre, H. Maillotte, J. Monneret, J. Opt. Soc. Am. B 14 (1) (1997) 116

[20] J.C. Boggio, J. Marconi, H. Fragnito, IEEE Photon. Technol. Lett. $18(2005) 1842$.

[21] F. Matera, M. Settembre, IEEE J. Lightwave Technol. 14 (1996) 1.

[22] M. Karlsson, J. Opt. Soc. Am. B 15 (8) (1998) 2269.

[23] A. Durécu-Legrand, C. Simonneau, D. Bayart, A.Mussot, E. Lantz, T. Sylvestre, H. Maillotte, Impact of the longitudinal variations of the chromatic dispersion on the gain of fiber parametric amplifiers, in: Optical Amplifiers and their Applications, No. WD2, Otaru, Japan, 2003.

[24] A. Mussot, E. Lantz, A. Durécu-Legrand, C. Simonneau, D. Bayart, T. Sylvestre, H. Maillotte, IEEE Photon. Technol. Lett. 18 (2006) 22. 